This article was downloaded by:

On: 3 September 2009

Access details: Access Details: Free Access

Publisher Taylor \& Francis

Informa Ltd Registered in England and Wales Registered Number: 1072954 Registered office: Mortimer House, 37-41 Mortimer Street, London W1T 3JH, UK

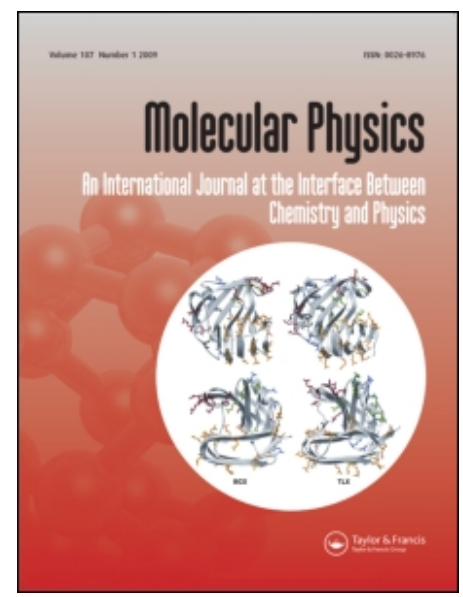

Molecular Physics

Publication details, including instructions for authors and subscription information:

http://www.informaworld.com/smpp/title content=t713395160

\title{
On nuclear magnetic relaxation due to slow molecular reorientation and fast
} internal rotation interference

Lian-Pin Hwang ab

a Department of Chemistry, National Taiwan University, Taipei, Taiwan, Republic of China ${ }^{\mathrm{b}}$ Institute of Biological Chemistry, Academia Sinica, Nankang, Taipei, Taiwan, Republic of China

Online Publication Date: 20 August 1983

To cite this Article Hwang, Lian-Pin(1983)'On nuclear magnetic relaxation due to slow molecular reorientation and fast internal rotation interference',Molecular Physics,49:6,1341 - 1352

To link to this Article: DOI: $10.1080 / 00268978300101991$

URL: http://dx.doi.org/10.1080/00268978300101991

\section{PLEASE SCROLL DOWN FOR ARTICLE}

Full terms and conditions of use: http://www.informaworld.com/terms-and-conditions-of-access.pdf

This article may be used for research, teaching and private study purposes. Any substantial or systematic reproduction, re-distribution, re-selling, loan or sub-licensing, systematic supply or distribution in any form to anyone is expressly forbidden.

The publisher does not give any warranty express or implied or make any representation that the contents will be complete or accurate or up to date. The accuracy of any instructions, formulae and drug doses should be independently verified with primary sources. The publisher shall not be liable for any loss, actions, claims, proceedings, demand or costs or damages whatsoever or howsoever caused arising directly or indirectly in connection with or arising out of the use of this material. 
Molecular Physics, 1983, Vol. 49, No. 6, 1341-1352

\title{
On nuclear magnetic relaxation due to slow molecular reorientation and fast internal rotation interference
}

\author{
by LIAN-PIN HWANG \\ Department of Chemistry, National Taiwan University, \\ Taipei, Taiwan, Republic of China, \\ and Institute of Biological Chemistry, Academia Sinica, \\ Nankang, Taipei, Taiwan, Republic of China
}

(Received 9 December 1982 ; accepted 23 March 1983)

\begin{abstract}
Spin-lattice relaxation arising from modulation of the dipole-dipole interaction by slow molecular motion together with fast internal rotation interference is investigated. The magnetic interaction for the internal rotation is treated as a perturbation and a modified stochastic Liouville equation, which is exact in all orders of the residual perturbation for the overall reorientation of the molecule, is derived after averaging over the internal rotation modes. The resultant equation is solved by the eigenfunction expansion method to study the spin-lattice relaxation. Results show complicated damping oscillations which Donskaya's work has failed to predict. Such oscillations in the relaxation are found in the slow motion region. Effects due to jump diffusion and brownian diffusion in the internal rotation are also considered.
\end{abstract}

\section{INTRODUCTION}

For a complex molecule, internal motions and overall rotation are present simultaneously, so that the correlation time relevant to relaxation may be a composite of the individual times. In 1962, Woessner [1] derived a theory for isolating methyl group rotation from overall rotation on the basis of second order perturbation theory by considering small dipolar interactions and fast rotational motions. Several years ago, Donskaya [2] presented a theory for nuclear magnetic dipolar relaxation due to slow molecular reorientation and fast internal rotation interference. The starting point was based on the observation that the consideration of the relaxation processes with the help of the ordinary Bloch equations is inadequate since the condition of the fast motion is not fulfilled by the slow reorientation motion of the molecule. Thus, the so called 'Woessner equation' for dipolar relaxation with internal motion is inapplicable to this case. In Donskaya's theory, a longitudinal relaxation rate which is of molecular orientation dependence is incorporated into the stochastic Liouville equation (SLE) which contains the prescribed relaxation part and the term describing the diffusion change of the magnetization due to overall reorientation of the molecule, e.g.,

$$
\frac{d M_{z}(\Omega, t)}{d t}=-\frac{M_{z}(\Omega, t)-M_{z}{ }^{0}(\Omega)}{T_{1}(\Omega)}-\Gamma_{\Omega}\left(M_{z}(\Omega, t)-M_{z}{ }^{0}(\Omega)\right),
$$


with

$$
\Gamma_{\Omega}=\Gamma_{\theta}=R\left(\frac{\partial^{2}}{\partial \theta^{2}}+\cot \theta \frac{\partial}{\partial \theta}\right),
$$

where $R$ is the rotational diffusion coefficient and $\theta$ is the polar angle between the molecular reorientational axis and the direction of the Zeeman field. Furthermore, we consider the case in which the proton-proton dipolar interaction has a constant separation distance $r$ while the internuclear vector rotates inside the molecule with a constant angle $\beta$ between the internuclear vector and the molecular $z$-axis. We may assume that this fast internal rotational motion occurs independently of the slow overall reorientational motion. In the limits of small dipolar perturbation and fast motion, i.e., $\omega_{I} \tau \ll 1$, where $\omega_{I}$ is the Larmor frequency of proton, the molecular orientation-dependent spin-lattice relaxation rate, at the instant of molecular orientation specified by the Eulerian angle $\Omega$, gives

with

$$
T^{-1}(\Omega)=a_{0}^{2} \tau\left[\eta_{0}(\beta)+\eta_{2}(\beta) \cos ^{2} \theta\right]
$$

and

$$
\eta_{0}(\beta)=\frac{9}{32}\left[5 \sin ^{2} 2 \beta+2\left(\tau_{2} / \tau\right) \sin ^{4} \beta\right]
$$

$$
\eta_{2}(\beta)=\frac{27}{32}\left[2\left(\tau_{2} / \tau\right) \sin ^{4} \beta-\sin ^{2} 2 \beta\right],
$$

where $\tau$ and $\tau_{2}$ are the correlation times for internal rotation, with $\tau_{2}=\tau$ for $120^{\circ}$-jump model and $\tau_{2}=\tau / 4$ for the brownian diffusion model $[3,4] . a_{0} \equiv$ $\gamma_{I} \gamma_{I}, r^{3}$ is the strength of proton dipolar interaction. However, if we examine equation (1), the residual dipolar interaction, after averaging over internal rotational modes, is missing in the expression and it is important to note that the missing term plays the main role in spin relaxation. It may be seen from the fact that as we take $\beta=0$ the first term on the right hand side of (1) drops out. As a result, the system does not show spin-relaxation.

A recent study of the spectral effects of combined motions has been made by Campbell et al. [10], who applied SLE to the study of N.M.R. lineshapes in a system of a decoupled $I=\frac{1}{2}$ nucleus with relatively rapid internal rotation and slow overall motions. In this paper we develop a model which, as compared with those already existing, offers a more realistic physical description for slow overall reorientation with internal rotation as a whole. Our main interest is in interpreting the magnetic relaxation in the slow motion region. Results of this theory are compared with those developed previously.

\section{ReLAXATION THEORY}

In our approach we have utilized the stochastic Liouville equation and considered independent motions between overall reorientation and internal rotation, i.e.

$$
\dot{\rho}(\Omega, \alpha, t)=\left(-i \mathscr{L}-\Gamma_{\Omega}-\Gamma_{\alpha}\right) \rho(\Omega, \alpha, t),
$$

where $\rho(\Omega, \alpha, t)$ is the spin density matrix in a state $(\Omega, \alpha)$ where $\alpha$ is the internal rotation angle. $\Gamma_{\alpha}$ and $\Gamma_{\Omega}$ are the diffusion operators for internal rotation and overall reorientation respectively. Upon Fourier transformation it yields

$$
\left(\omega+\mathscr{L}-i \Gamma_{\Omega}-i \Gamma_{\alpha}\right) \rho[\Omega, \alpha, \omega]=-i \rho(\Omega, \alpha, 0),
$$


where $\mathscr{L}$ is the Liouville operator corresponding to the total spin hamiltonian $H$

$$
\mathscr{L} \equiv[H, \quad] \equiv \mathscr{L}_{0}+\mathscr{L}_{1}(\Omega, \alpha) .
$$

Here $\mathscr{L}_{0}$ is the Liouville operator for the Zeeman term and $\mathscr{L}_{1}(\Omega, \alpha)$ is the perturbation Liouville operator depending on the orientation $\Omega$ and internal rotation coordinates $(\alpha, \beta, 0)$. Its corresponding hamiltonian may be expressed as the scalar product of two tensors. That is we write $H_{1}(\Omega, \alpha)$ as

$$
\begin{aligned}
H_{1}(\Omega, \alpha) & =\sum_{p, q} D_{q, 0}{ }^{(2)}(\alpha, \beta, 0) D_{q, p}{ }^{(2)}(\Omega) A_{p} \\
& =\sum_{p, q} \exp (i q \alpha) d_{q, 0}{ }^{(2)}(\beta) D_{q, p}{ }^{(2)}(\Omega) A_{p},
\end{aligned}
$$

where $D_{q, p}{ }^{(2)}(\Omega)$ and $d_{q, 0}{ }^{(2)}(\beta)$ are the usual and the reduced Wigner rotational matrix elements respectively, and where for dipolar interaction of nuclear spins $I$ and $I^{\prime} A_{p}$ is given by

$$
\begin{aligned}
& A_{2}=\sqrt{ } \frac{b}{3} \frac{b}{r^{3}} I_{+} I_{+}^{\prime} \\
& A_{1}=-\sqrt{ } \frac{b}{3} \frac{b}{r^{3}}\left(I_{z} I_{+}^{\prime}+I_{+} I_{z}^{\prime}\right) \\
& A_{0}=\frac{-2 b}{r^{3}}\left(\frac{-2}{3} I_{z} I_{z}^{\prime}+\frac{1}{6} I_{+} I_{-}+\frac{1}{6} I_{-} I_{+}^{\prime}\right) \\
& A_{-1}=\sqrt{ } \frac{2}{3} \frac{b}{r^{3}}\left(I_{z} I_{-}^{\prime}+I_{-} I_{z}^{\prime}\right) \\
& A_{-2}=\sqrt{ } \frac{b}{3} \frac{b}{r^{3}} I_{-} I_{-}^{\prime}
\end{aligned}
$$

with

$$
b \equiv(3 / 2) \gamma_{I} \gamma_{I}, \hbar
$$

We now wish to simplify the SLE $[7,8]$ to obtain a distribution function for just the orientational space of the molecule. Since $\alpha$ is a fast variable and the internal rotation and the overall reorientation are assumed independent we may introduce a coarse graining in time such that the spin density matrix is averaged over times $t_{\mathrm{c}} \gg \tau\left(\right.$ or $\tau_{2}$ ) but still small compared to $R^{-1}$. Then, such a coarse graining in time gives the expression for $\rho$ in terms of a complete orthogonal set of eigenfunctions.

$$
\rho(\Omega, \alpha, t) \equiv \sum_{n} \sigma_{n}(\Omega, t)\left|G_{n}(\alpha)\right\rangle,
$$

where we have $\Gamma_{\alpha}\left|G_{n}(\alpha)\right\rangle=1 / \tau_{n}\left|G_{n}(\alpha)\right\rangle$ and generally let $G_{0}(\alpha)=(1 / 2 \pi)^{1 / 2}$ which corresponds to the equilibrium distribution in $\alpha$ space. $\sigma_{n}(\Omega, t)$ is the spin density associated with a particular value of $\Omega$ and the $n$th internal rotational state. The equation of motion on the time scale associated with $t_{c}$ may be obtained by defining a projection operator such as

or

$$
\hat{p} \rho(\Omega, \alpha, t) \equiv \sigma_{0}(\Omega, t),
$$

$$
\hat{p} \equiv\left\langle G_{0}(\alpha)\right| .
$$


Operating with $\hat{p}$ and $(1-\hat{p})$ on the SLE respectively, we obtain

$$
\begin{aligned}
\left(\omega+\mathscr{L}_{0}+\mathscr{L}_{a}-i \Gamma_{\Omega}\right) \sigma_{0}[\Omega, \omega]+\hat{p} \mathscr{L}_{1}(1-\hat{p}) \rho[\Omega, \alpha, \omega] & =-i \sigma_{0}(\Omega, 0), \\
\left(\omega+\mathscr{L}_{0}+(1-\hat{p}) \mathscr{L}_{1}-i \Gamma_{\Omega}-i \Gamma_{\alpha}\right)(1-\hat{p}) \rho[\Omega, \alpha, \omega] & =\left(\mathscr{L}_{d}-\mathscr{L}_{1}\right) \sigma_{0}[\Omega, \omega],
\end{aligned}
$$

where

$$
\mathscr{L}_{d} \sigma_{0} \equiv\left[H_{d}, \sigma_{0}\right]=\hat{p} \mathscr{L}_{1} \sigma_{0} .
$$

It is noted that $\Gamma_{\alpha}(1-\hat{p}) \rho$ does not vanish so that this term suffices to ensure the fast motion condition, $\left(H_{1}-H_{d}\right) \tau_{n} \ll 1$. We may simplify equations (14) in analogy with the usual second-order perturbation theory and obtain

$$
\begin{aligned}
\left(\omega+\mathscr{L}_{0}+\mathscr{L}_{d}-i \Gamma_{\Omega}\right) \sigma_{0}[\Omega, \omega]+\hat{p} \mathscr{L}_{1}\left(\omega+\mathscr{L}_{0}-i \Gamma_{\Omega}-i \Gamma_{\alpha}\right)^{-1} \\
\times\left(\mathscr{L}_{d}-\mathscr{L}_{1}\right) \sigma_{0}[\Omega, \omega]=-i \sigma_{0}(\Omega, 0) .
\end{aligned}
$$

The second term on the left may be simplified further to give

$$
\left(\omega+\mathscr{L}_{0}+\mathscr{L}_{d}-i \Gamma_{\Omega}-i K[\omega]\right) \sigma_{0}[\Omega, \omega]=-i \sigma_{0}(\Omega, 0),
$$

where we define

$$
K[\omega]=\frac{1}{2 \pi} \int_{0}^{2 \pi} d \alpha\left(\mathscr{L}_{1}-\mathscr{L}_{d}\right)\left(i \omega+i \mathscr{L}_{0}+\Gamma_{\Omega}+\Gamma_{\alpha}\right)^{-1}\left(\mathscr{L}_{1}-\mathscr{L}_{d}\right) .
$$

If we transform (17) back to time space we find

$$
\frac{\partial \sigma_{0}(\Omega, t)}{\partial t}=-i\left(\mathscr{L}_{0}+\mathscr{L}_{d}\right) \sigma_{0}(\Omega, t)-\Gamma_{\Omega} \sigma_{0}(\Omega, t)-\int_{0}^{t} K\left(t-t_{1}\right) \sigma_{0}\left(\Omega, t_{1}\right) d t_{1} .
$$

It is important to notice that the kernel $K(t)$ is the memory function [6] which describes the magnetic relaxation due to the internal fast processes. Since the internal rotational mode is considered $a$ priori to be relatively much more rapid than the overall reorientation, $K(t)$ may decay appreciably while $\sigma_{0}(\Omega, t)$ remains almost unchanged. Thus, it seems quite satisfactory to single out the relaxation process arising from the internal rotation mode. So we obtain

where

$$
\dot{\sigma}_{0}(\Omega, t)=\left[-i\left(\mathscr{L}_{0}+\mathscr{L}_{a}\right)-\Gamma_{\Omega}-R\right] \sigma_{0}(\Omega, t),
$$

$$
\mathcal{R}=\int_{0}^{\infty} K(t) d t \simeq \frac{1}{2 \pi} \int_{0}^{\infty} d t \int_{0}^{2 \pi} d \alpha\left(\mathscr{L}_{1}-\mathscr{L}_{d}\right) \exp \left(-i \mathscr{L}_{0} t-\Gamma_{\alpha} t\right)\left(\mathscr{L}_{1}-\mathscr{L}_{d}\right) .
$$

This is the stochastic Liouville equation for the present model. An alternative to this derivation may be obtained by invoking the van Vleck method [11] in conjunction with the approach outlined in the Appendix of [10]. We now note that the first term in (19a) gives the dynamical motion of the spin degrees of freedom preaveraged over internal modes. It was missing in Donskaya's formulation (cf. equation (1)), while, for spin-lattice relaxation, the second term has its usual meaning as described in his equation except that axially symmetric diffusion is considered in this work. For a two spin system of identical spins $\left(I=\frac{1}{2}\right)$ with dipolar interaction and fast internal rotation, the third term in $(19 a)$, after some simple manipulation [5] may be identified as $1 / T_{1}(\Omega)$ in Donskaya's formulation. Moreover, the second and third terms have real parts which are 
responsible for relaxation. In the rigorous treatment of the stochastic Liouville equation in the limit as $t \rightarrow \infty, \sigma_{0}(\Omega, t)$ may achieve its equilibrium value $\sigma_{\text {eq }}(\Omega)$ by including a 'spin torque' term which has the property of tending to restore the spins to their thermal equilibrium value [7]. However, for convenience, and also for retaining the physical significance, one may have an ad hoc assumption by substituting

$$
\sigma(\Omega, t) \equiv \sigma_{0}(\Omega, t)-\sigma_{\text {eq }}(\Omega)
$$

for $\sigma_{0}(\Omega, t)$ in SLE [7]. Here $\sigma_{\mathrm{eq}}(\Omega)$ is the equilibrium density matrix whose $\Omega$ dependence is $\Gamma_{\Omega} \sigma_{\text {eq }}(\Omega)=0$ in order to assure the solution approaching $\sigma_{\text {eq }}$ in the limit as $t \rightarrow \infty$. For isotropic distributions we have $\sigma_{\text {eq }} \equiv\left(\int d \Omega\right)^{-1}$. Quantities of interest in the calculation may be expressed by

$$
\begin{aligned}
M_{z}(t)-M_{z}{ }^{0} & =\left\langle\left(\left(I_{z}+I_{z}^{\prime}\right) \sigma(\Omega, t)\right)\right\rangle \\
& =\frac{1}{8 \pi^{2}} \int d \Omega \frac{1}{4}(\langle++|\sigma(\Omega, t)|++\rangle-\langle--|\sigma(\Omega, t)|--\rangle)
\end{aligned}
$$

where $M_{z}^{0}$ is the equilibrium value of $z$ component magnetization. Then, it is clear that from the knowledge of the population difference between $|++\rangle$ and $\mid-->$ states, one may deduce the rate of the spin-lattice relaxation. This may be solved by using the expansion method due to Freed et al. [8] in their treatment of E.S.R. lineshapes for calculating $\left(M_{z}(t)-M_{z}\right)^{0}$ from SLE (19), for example

$$
\sigma(\Omega, t)=\sum_{L, K, M} C_{K, M}{ }^{L} D_{K, M}{ }^{L}(\Omega)\left[\left(8 \pi^{2}\right)^{-1}(2 L+1)\right]^{1 / 2} .
$$

The Wigner rotation matrices $D_{K, \mu^{L}}(\Omega)$ with eigenvalues $E_{K, M^{L}}=\tau_{L, K}{ }^{-1}$, as given by

$$
\tau_{L, K}{ }^{-1}=R_{+} L(L+1)+\left(R_{z}-R_{+}\right) K^{2}
$$

provide such a set for axially symmetric brownian reorientational diffusion. Also, $R_{z}$ is the rotational diffusion coefficient about the molecular $z$-axis and $R_{+}$is the rotational diffusion coefficient about the axes perpendicular to the $z$ axis. Note that the difference in $z$-component magnetization with its equilibrium value may be expressed by

$$
\begin{aligned}
\left(M_{z}(t)-M_{z}^{0}\right) & =\frac{1}{4}\left[\left\langle++\left|C_{0,0}{ }^{0}\right|++\right\rangle-\left\langle--\left|C_{0,0}{ }^{0}\right|--\right\rangle\right] \\
& \equiv \frac{1}{4}\left[C_{0,0}{ }^{0}(a)-C_{0,0}{ }^{0}(b)\right],
\end{aligned}
$$

where $a$ and $b$ are the spin states shown in figure 1. Then, we obtain a hierarchy of equations of the types given in the Appendix pertaining to the spin-lattice relaxation process. In Appendix A, equations (A 1), (A 2) and (A 9) can be decoupled from (A 3)-(A 8) in the limit $\left|\omega_{I} \tau_{L, K}\right| \gg 1,[8,11]$ which is appropriate for slow motions. However, to treat the problems in general, the infinite set of equations may be written in matrix notation as

$$
\dot{C}=A C \text {, }
$$

where $C$ is a column vector of all the matrix elements of the coefficients $C_{K}, x^{L}$, while $A$ is seen from (A 1)-(A 9) to be a complex symmetric matrix, which may be recast into $\mathrm{A} \equiv \mathbf{A}^{\prime}-\mathbf{B I}$ with $(B)_{i} \equiv B \equiv a_{0}{ }^{2} \tau\left(\eta_{0}(\beta)+\frac{1}{3} \eta_{2}(\beta)\right)$. Equation (25) 


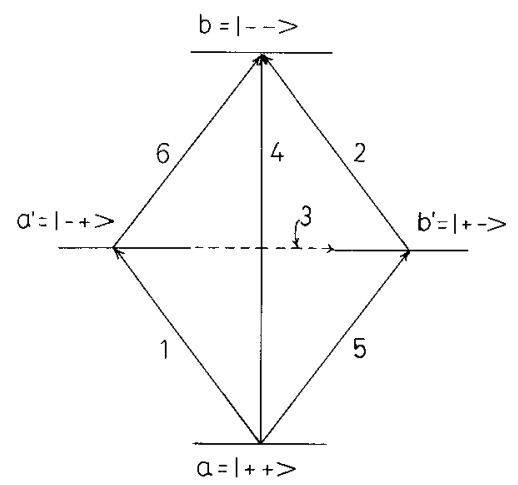

Figure 1. Energy level diagram for combined spin states of two coupled protons. Numbers $1,2,3,4,5$ and 6 identify relaxation transitions.

is solved in the standard manner [8] by finding a complex orthogonal matrix $\mathbf{S}$ to diagonalize $\mathbf{A}^{\prime}$ such as $\mathbf{S}^{-1} \mathbf{A}^{\prime} \mathbf{S}=\boldsymbol{\Lambda}$. Then we obtain the solution

$$
\mathrm{C}=\mathrm{SES}^{-1} \mathrm{C} t_{0}
$$

where the diagonalized matrix $\mathbf{E}$ is defined by

$$
\mathbf{E}_{i}=\exp \left(-\left(\boldsymbol{\Lambda}_{i i}+B\right) t\right),
$$

and where $\mathbf{C}_{t_{0}}=\mathbf{C}_{\left.\right|_{t=0}}$ is a column matrix related to the initial distribution of the matrix elements in $\mathbf{C}$. The specific form of $\mathbf{C}_{t_{0}}$ depends on the method of creation of the initial non-equilibrium magnetization. To compare with Donskaya's results, we utilize the initial condition, $\left(M_{z}(0)-M_{z}^{0}\right)=-M_{z}^{0}$, for the magnetization after a $90^{\circ}$ pulse.

In this case, we take

$$
\begin{aligned}
& \left(C_{t_{0}}\right)_{1} \equiv\left(C_{0,0}{ }^{0}(a)\right)_{t_{0}} \simeq\left(1-\exp \left(\gamma_{I} \hbar H_{0} / k T\right)\right) \cdot m, \\
& \left(C_{t_{0}}\right)_{2} \equiv\left(C_{0,0}{ }^{0}(b)\right)_{t_{0}} \simeq\left(1-\exp \left(-\gamma_{I} \hbar H_{0} / k T\right)\right) \cdot m,
\end{aligned}
$$

where $H_{0}$ is the Zeeman field and $m$ stands for the total magnetic moment. Note that other column matrix elements are identically zero as the initial preparation of the system. The diagonalization scheme leads itself to the computation of the time-evolution of $\left(M_{z}(t)-M_{z}^{0}\right)$

$$
\left(M_{z}(t)-M_{z}^{0}\right)=\frac{1}{4} \exp (-B t) \sum_{j, k}\left(\mathbf{S}_{1 j}-\mathbf{S}_{2 j}\right) \exp \left(-\boldsymbol{\Lambda}_{j j} t\right)(\mathbf{S})_{j k}\left(\mathbf{C}_{t_{0}}\right)_{k}
$$

which describes the approach of $M_{z}(t)$ to the equilibrium value.

\section{Results AND Discussion}

In the procedure of diagonalization, convergent solutions may be obtained by terminating the expansion of orthogonal functions (cf. equation (22)) at a 
sufficiently large value of $L$. For the cases studied for ${a_{0}}^{2} \tau / R_{+} \ll 1$, the magnetization decays exponentially. The $T_{1}$ values are obtained by the least square fitting of $\left(M_{z}(t)-M_{z}^{0}\right)$ vs. $\ln (t)$ with correlation coefficients better than 0.998 . The variation of $T_{1}$ with respect to $a_{0} / R_{+}$for brownian diffusion and jump diffusion are shown in figures 2 and 3 , respectively, where $a_{0}=\gamma_{I} \gamma_{I}, r^{3}$ is the strength of proton dipolar interaction. The angles between the internuclear vector and the molecular $z$ axis is set to equal $\pi / 2$. It is observed, from the figures, that the Donskaya model gives constant values in this region and fails to show the effect of change of motion. For comparison, we deliberately calculate $T_{1}$ from Woessner's equation which is based on the second order perturbation theory and is invalid in the region where $a_{0} / R_{+}>1$. However, the result from Woessner's equation achieves excellent agreement with the present calculation

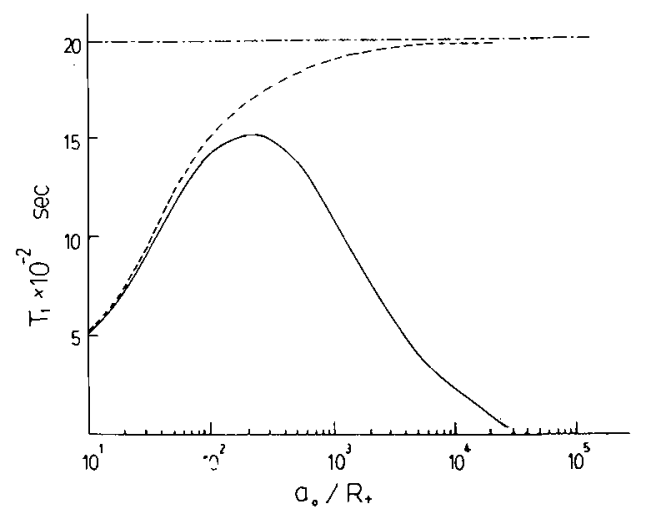

Figure 2. Calculation of $60 \mathrm{MHz}$ proton $T_{1}$ versus $a_{0} / R_{+}$for proton-proton dipolar relaxation with $r=1.78 \AA$ and $\beta=90^{\circ}$ in a methyl group based upon the brownian rotational diffusion with $\tau=1 \times 10^{-13} \mathrm{~s}$. The solid curve is obtained in this work. The dashed curve is the result of Woessner, while the broken line is the result of Donskaya, $R_{+}=R_{z}$ is assumed in the calculation.

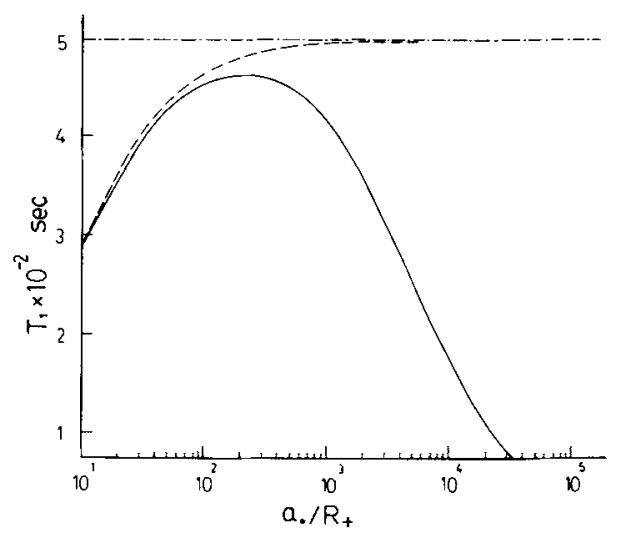

Figure 3. Calculation of $T_{1}$ versus $a_{0} / R_{+}$for proton-proton dipolar relaxation in a methyl group based upon the jump diffusion model. Curve description and parameters used are the same as given in figure 2. 
even for $a_{0} / R_{+}$as large as 10 . The main difference between Donskaya's results and present theory is in the preaveraged dipolar term. This has the effect of significantly reducing the magnitude of $T_{1}$ predicted in the region $a_{0} / R_{+}>1$. Furthermore, the $T_{1}$ value obtained from Woessner's equation begins to increase and reaches an asymptotic value $B^{-1}$ as indicated in Donskaya's model. The difference between our model and Woessner's result becomes less pronounced at higher magnetic field. As can be seen from figures 2 and 3 the spin-lattice relaxation times are rather sensitive to the diffusion model used. Also, the brownian diffusion model yields longer $T_{1}$ values than does the jump diffusion model.

For a molecule undergoing anisotropic rotational diffusion, one may expect that the effective diffusion coefficient parallel to the molecular axis may be interpreted as $1 / \tau$ while the overall motion may be represented by a weighted average of $R_{z}$ and $R_{+}$, that depends upon the angle, $\beta$, chosen. For the cases demonstrated in figures 2 and 3 , if $R_{z} \neq R_{+}$and $R_{z} \ll 1 / \tau$ the results are unaffected by the variation of $R_{z}$ and all motional effects are characterized by $R_{+}$and $1 / \tau$ $\left(1 / \tau_{2}\right)$. The conclusion is parallel to the result obtained by Campbell et al. [10].

In figure 4, we show the slow motion effects on $M_{z}(t)$ as $a_{0}{ }^{2} \tau / R_{+} \gg 1$. Even at the initial stage, it is observed that the decay of $\left(M_{z}(t)-M_{z}^{0}\right)$ follows neither a single exponential function nor a composite two-exponential-function as predicted by Donskaya, but complex damping oscillations are obtained. This is characteristics of a non-markoffian relaxation effect due to the modulation by slow reorientation motion of a magnetic interaction when the motion beeomes slow $\left(H_{1}{ }^{2} \tau / R_{+}>1\right)$. It can be seen that the curve corresponding to $\beta=90^{\circ}$, shows stronger damping than the curve with $\beta=45^{\circ}$. This may be interpreted in terms of a larger magnitude of magnetic interaction $\left(\eta_{0}(\beta)+\eta_{2}(\beta)\left\langle\cos ^{2} \theta\right\rangle\right)$ for the case with $\beta=90^{\circ}$. As the motion of overall reorientation gets slower the results show only a slight influence on the behaviour of $M_{z}(t)$ at times $t<R_{+}{ }^{-1}$. Also, it is interesting to know that in contrast to the results found for $a_{0}{ }^{2} \tau / R_{+} \ll 1$, the relaxation processes for those cases with $a_{0}{ }^{2} \tau / R_{+} \gtrsim 1$ are insensitive to the details of diffusion model chosen for internal rotation.

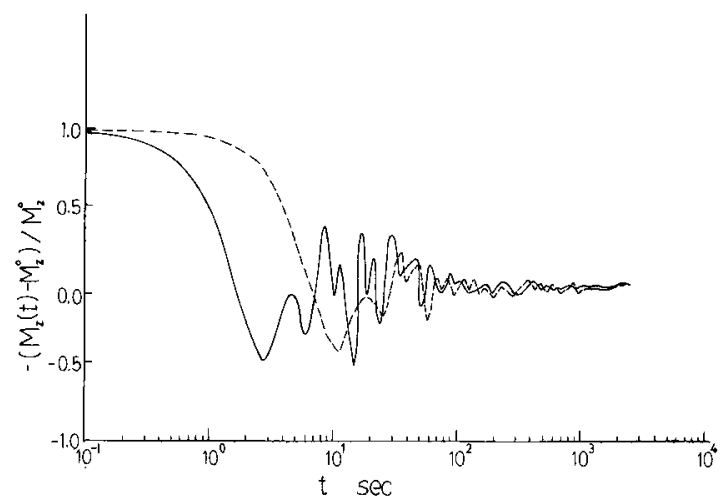

Figure 4. Plot of $\left(M_{z}^{0}-M_{z}(t)\right) / M_{z}^{0}$ versus time for proton-proton dipolar relaxation in a methyl group based upon the jump diffusion and the overall reorientational diffusion coefficient $R_{+}=R_{z}=10^{-3} \mathrm{~s}^{-1}$. The dashed curve is for $\beta=45^{\circ}$ while the solid curve for $\beta=90^{\circ}$. Other parameters are the same as given in figure 2 . 


\section{ConClusion}

The purpose of this paper has been to formulate tractable models for molecules with slow overall reorientation and fast internal rotation. Of particular significance is that the missing term in the previous theory is effective in causing relaxation. There is considerable current interest in the nature of the molecular motion in macromolecules. Thus, the modified SLE may provide sufficient theoretical framework so that models considered here can readily be applied and extended to the study of relaxation process of a nucleus in a side chain, containing multiple internal rotations, which is attached to a large macromolecule [9].

We thank the National Science Council of the Republic of China for support of this work.

\section{APPENDIX}

The general equations which define the time-dependent magnetization for dipolar proton-proton interaction are given by

$$
\begin{aligned}
& C_{0,0} L^{L}(a)=-i A d_{0,0}{ }^{2}(\beta) \sum_{L^{\prime}} N\left(L, L^{\prime}\right) \times\left(\begin{array}{ccc}
L & 2 & L^{\prime} \\
0 & 0 & 0
\end{array}\right)\left[\left(\begin{array}{ccc}
L & 2 & L^{\prime} \\
0 & 2 & -2
\end{array}\right)\right. \\
& \times C_{0,-2}{ }^{L^{\prime}}(4)-\frac{1}{\sqrt{ } 2}\left(\begin{array}{ccc}
L & 2 & L^{\prime} \\
0 & 1 & -1
\end{array}\right) C_{0,-1}{ }^{\prime}(5,1)-\frac{1}{\sqrt{ } 2} \\
& \times\left(\begin{array}{ccc}
L & 2 & L^{\prime} \\
0 & -1 & 1
\end{array}\right) C_{0,1} L^{\prime}(-5,-1)-\left(\begin{array}{ccc}
L & 2 & L^{\prime} \\
0 & -2 & 2
\end{array}\right) \\
& \left.\times C_{0,2}{ }^{L}(-4)\right]-\left[1 / \tau_{L, 0}+B+C(L, L ; 0,0)\right] C_{0,0}{ }^{L}(a) \\
& -C(L, L-2 ; 0,0) C_{0,0}{ }^{L-2}(a) \\
& -C(L, L+2 ; 0,0) C_{0,0}{ }^{L+2}(a) \text {, } \\
& C_{0,0}{ }^{L}(b)=-i A d_{0,0}{ }^{2}(\beta) \sum_{L^{\prime}} N\left(L, L^{\prime}\right) \times\left(\begin{array}{ccc}
L & 2 & L^{\prime} \\
0 & 0 & 0
\end{array}\right)\left[-\left(\begin{array}{ccc}
L & 2 & L^{\prime} \\
0 & 2 & -2
\end{array}\right)\right. \\
& \times C_{0,-2} L^{\prime}(4)-\frac{1}{\sqrt{ } 2}\left(\begin{array}{ccc}
L & 2 & L^{\prime} \\
0 & 1 & -1
\end{array}\right) C_{0,-1} L^{\prime}(6,2)-\frac{1}{\sqrt{ } 2} \\
& \times\left(\begin{array}{ccc}
L & 2 & L^{\prime} \\
0 & -1 & 1
\end{array}\right) C_{0,1} L^{\prime}(-6,-2)+\left(\begin{array}{ccc}
L & 2 & L^{\prime} \\
0 & 2 & -2
\end{array}\right) \\
& \left.\times C_{0,2}{ }^{L^{\prime}}(-4)\right]-\left[1 / \tau_{L, 0}+B+C(L, L ; 0,0)\right] C_{0,0}{ }^{L}(b) \\
& -C(L, L-2 ; 0,0) C_{0,0}{ }^{L-2}(b) \\
& -C(L, L+2 ; 0,0) C_{0,0}{ }^{L+2}(b),
\end{aligned}
$$




$$
\begin{aligned}
& \dot{C}_{0,-2} L(4)=-i A d_{0,0}^{2}(\beta) \sum_{L^{\prime}} N\left(L, L^{\prime}\right)\left(\begin{array}{ccc}
L & 2 & L^{\prime} \\
0 & 0 & 0
\end{array}\right)\left[-\frac{1}{\sqrt{ } 2}\right. \\
& \times\left(\begin{array}{ccc}
L & 2 & L^{\prime} \\
2 & -1 & -1
\end{array}\right)\left(C_{0,-1} L^{\prime}(5,1)+C_{0,-1} L^{\prime}(6,2)\right) \\
& \left.+\left(\begin{array}{ccc}
L & 2 & L^{\prime} \\
2 & -2 & 0
\end{array}\right)\left(C_{0,0} L^{\prime}(a)-C_{0,0} L^{\prime}(b)\right)\right] \\
& -\left[2 i \omega_{I}+1 / \tau_{L,-2}+B+C(L, L ; 0,-2)\right] C_{0,-2}{ }^{L}(4) \\
& \text { - } C(L, L-2 ; 0,-2) C_{0,-2}^{L-2}(4) \\
& -C(L, L+2 ; 0,-2) C_{0,-2}^{L+2}(4) \text {, } \\
& \dot{C}_{0,-1} L(5,1)=-i A d_{0,0}{ }^{2}(\beta) \sum_{L^{\prime}} N\left(L, L^{\prime}\right)\left(\begin{array}{ccc}
L & 2 & L^{\prime} \\
0 & 0 & 0
\end{array}\right)\left[-\frac{1}{\sqrt{ } 2}\left(\begin{array}{ccc}
L & 2 & L^{\prime} \\
1 & 1 & -2
\end{array}\right)\right. \\
& \times C_{0,-2} L^{\prime}(4)+\sqrt{ }\left(\frac{3}{2}\right)\left(\begin{array}{ccc}
L & 2 & L^{\prime} \\
1 & 0 & -1
\end{array}\right) C_{0,-1} L^{\prime}(5,1)-\frac{1}{\sqrt{ } 2} \\
& \times\left(\begin{array}{ccc}
L & 2 & L^{\prime} \\
1 & -1 & 0
\end{array}\right)\left(C_{0,0} L^{\prime}(a)-C_{0,0} L^{\prime}(m)\right) \\
& \left.+\left(\begin{array}{ccc}
L & 2 & L^{\prime} \\
1 & -2 & 1
\end{array}\right) C_{0,1} L^{\prime}(-6,-2)\right] \\
& -\left[i \omega_{I}+1 / \tau_{L,-1}+B+C(L, L ; 0,-1)\right] C_{0,-1} L(5,1) \\
& -C(L, L-2 ; 0,-1) C_{0,-1}^{L-2}(5,1) \\
& -C(L, L+2 ; 0,-1) C_{0,-1}^{L+2}(5,1) \\
& C_{0,-1} L(6,2)=-i A d_{0,0}{ }^{2}(\beta) \sum_{L^{\prime}} N\left(C, L^{\prime}\right)\left(\begin{array}{ccc}
L & 2 & L^{\prime} \\
0 & 0 & 0
\end{array}\right)\left[-\frac{1}{\sqrt{ } 2}\right. \\
& \times\left(\begin{array}{ccc}
L & 2 & L^{\prime} \\
1 & 1 & -2
\end{array}\right) C_{0,-2} L^{\prime}(4)-\sqrt{ }\left(\frac{3}{2}\right)\left(\begin{array}{ccc}
L & 2 & L^{\prime} \\
1 & 0 & -1
\end{array}\right) \\
& \times C_{0,-1} L^{\prime}(6,2)-\frac{1}{\sqrt{ } 2}\left(\begin{array}{ccc}
L & 2 & L^{\prime} \\
1 & -1 & 0
\end{array}\right)\left(C_{0,0}{ }^{L^{\prime}}(b)\right. \\
& \left.\left.-C_{0,0} L^{\prime}(m)\right)-\left(\begin{array}{ccc}
L & 2 & L^{\prime} \\
1 & -2 & 1
\end{array}\right) C_{0,1} L^{\prime}(-5,-1)\right] \\
& -\left(i \omega_{I}+1 / \tau_{L,-1}+B+C(L, L ; 0,-1) C_{0,-1} L(6,2)\right. \\
& -C(L, L-2 ; 0,-1) C_{0,-1}{ }^{L-2}(6,2) \\
& -C(L, L+2 ; 0,-1) C_{0,-1}^{L+2}(6,2) \text {, }
\end{aligned}
$$




$$
\begin{aligned}
& C_{0,1} L^{2}(-5,-1)=-i A d_{0,0}{ }^{2}(\beta) \sum_{L^{\prime}} N\left(L, L^{\prime}\right)\left(\begin{array}{lll}
L & 2 & L^{\prime} \\
0 & 0 & 0
\end{array}\right)\left[-\left(\begin{array}{ccc}
L & 2 & L^{\prime} \\
-1 & 2 & -1
\end{array}\right)\right. \\
& \times C_{0,-1} L^{\prime}(6,2)-\frac{1}{\sqrt{ } 2}\left(\begin{array}{ccc}
L & 2 & L^{\prime} \\
-1 & 1 & 0
\end{array}\right)\left(C_{0,0} L^{\prime}(a)\right. \\
& \left.-C_{0,0} L^{\prime}(m)\right)-\sqrt{ }\left(\frac{3}{2}\right)\left(\begin{array}{ccc}
L & 2 & L^{\prime} \\
-1 & 0 & 1
\end{array}\right) C_{0,1} L^{\prime}(-5,-1) \\
& \left.-\frac{1}{\sqrt{ } 2}\left(\begin{array}{ccc}
L & 2 & L^{\prime} \\
-2 & -1 & 2
\end{array}\right) C_{0,2} L^{\prime}(-4)\right] \\
& -\left(-i \omega_{I}+1 / \tau_{L, 1}+B+C(L, L ; 0,1)\right) C_{0,1}{ }^{L}(-5,-1) \\
& -C(L, L-2 ; 0,1) C_{0,1}^{L-2}(-5,-1) \\
& -C(L, L+2 ; 0,1) C_{0,1}^{L+2}(-5,-1),
\end{aligned}
$$

$C_{0,1}{ }^{L}(-6,-2)=-i A d_{0,0}{ }^{2}(\beta) \sum_{L^{\prime}} N\left(L, L^{\prime}\right)\left(\begin{array}{ccc}L & 2 & L^{\prime} \\ 0 & 0 & 0\end{array}\right)\left[\left(\begin{array}{ccc}L & 2 & L^{\prime} \\ -1 & 2 & -1\end{array}\right)\right.$

$$
\times C_{0,-1} L^{\prime}(5,1)-\frac{1}{\sqrt{ } 2}\left(\begin{array}{ccc}
L & 2 & L^{\prime} \\
-1 & 1 & 0
\end{array}\right)\left(C_{0,0} L^{\prime}(b)\right.
$$$$
\left.-C_{0,0} L^{\prime}(m)\right)+\sqrt{ }\left(\frac{3}{2}\right)\left(\begin{array}{ccc}
L & 2 & L^{\prime} \\
-1 & 0 & 1
\end{array}\right) C_{0,1} L^{\prime}(-6,-2)
$$$$
\left.-\frac{1}{\sqrt{ } 2}\left(\begin{array}{ccc}
L & 2 & L^{\prime} \\
-1 & -1 & 2
\end{array}\right) C_{0,2} L^{\prime}(-4)\right]
$$$$
-\left[-i \omega_{I}+1 / \tau_{L, 1}+B+C(L, L ; 0,1)\right] C_{0,1} L(-6,-2)
$$$$
-C(L, L-2 ; 0,1) C_{0,1}^{L-2}(-6,-2)
$$$$
-C(L, L+2 ; 0,1) C_{0,1}^{L+2}(-6,-2) \text {, }
$$

$$
\begin{aligned}
C_{0,2}{ }^{L}(-4)=- & i A d_{0,0}{ }^{2}(\beta) \sum_{L^{\prime}} N\left(L, L^{\prime}\right)\left(\begin{array}{ccc}
L & 2 & L^{\prime} \\
0 & 0 & 0
\end{array}\right)\left[\left(\begin{array}{ccc}
L & 2 & L^{\prime} \\
-2 & 2 & 0
\end{array}\right)\right. \\
& \times\left(C_{0,0} L^{\prime}(b)-C_{0,0} L^{\prime}(a)\right)-\frac{1}{\sqrt{ } 2}\left(\begin{array}{ccc}
L & 2 & L^{\prime} \\
-2 & 1 & 1
\end{array}\right) \\
& \left.\times\left(C_{0,1} L^{\prime}(-5,-1)+C_{0,1} L^{\prime}(-6,-2)\right)\right] \\
& -\left[-2 i \omega_{I}+1 / \tau_{L, 2}+B+C(L, L ; 0,2)\right] C_{0,2}{ }^{L}(-4) \\
& -C(L, L-2 ; 0,2) C_{0,2}^{L-2}(-4) \\
& -C(L, L+2 ; 0,2) C_{0,2} L+2(-4)
\end{aligned}
$$




$$
\begin{aligned}
C_{0,0}{ }^{L}(m)=- & i A d_{0,0}{ }^{2}(\beta) \sum_{L^{\prime}} N\left(L, L^{\prime}\right)\left(\begin{array}{ccc}
L & 2 & L^{\prime} \\
0 & 0 & 0
\end{array}\right)\left[\frac{1}{\sqrt{ } 2}\right. \\
& \left.\times\left(\begin{array}{ccc}
L & 2 & L^{\prime} \\
0 & -1 & 1
\end{array}\right)\left(C_{0,1} L^{\prime}(-6,-2)+C_{0,1} L^{\prime}(-5,-1)\right)\right] \\
& -\left[\begin{array}{c}
\left.1 / \tau_{L, 0}+B+C(L, L ; 0,0)\right] C_{0,0}{ }^{L}(m) \\
-
\end{array}\right. \\
- & -C(L, L-2 ; 0,0) C_{0,0}{ }^{L-2}(m) \\
& -C(L, L+2 ; 0,0) C_{0,0}{ }^{L+2}(m),
\end{aligned}
$$

where $N\left(L, L^{\prime}\right) \equiv\left((2 L+1)\left(2 L^{\prime}+1\right)\right)^{1 / 2}$ and the coefficients in the above equations are defined as

$$
\begin{aligned}
C_{K, M}{ }^{L}(m) & \equiv \frac{C_{K, M}{ }^{L}\left(a^{\prime}\right)+C_{K, M}{ }^{L}\left(b^{\prime}\right)+C_{K, M}{ }^{L}(3,-3)}{2}, \\
C_{K, M}{ }^{L}(p, q) & \equiv \frac{1}{\sqrt{ } 2}\left(C_{K, M}^{L}(p)+C_{K, M}{ }^{L}(q)\right),
\end{aligned}
$$

with

$$
C_{K, M}{ }^{L}(p)=\left\langle j\left|C_{K, M} L\right| i\right\rangle \text { for } p=1 \text { to } 6,
$$

where we denote the transition $|i\rangle$ to $|j\rangle$ as $p$ and the reverse transition as $-p$. The details of transitions can be found in figure 1. Furthermore $C_{K, M} L(a)$, $C_{K, M}{ }^{L}(b), C_{K, M}\left(a^{\prime}\right)$ and $C_{K, M}{ }^{L}\left(b^{\prime}\right)$ are the matrix elements of states $a, b, a^{\prime}$ and $b^{\prime}$ respectively. Definition of other terms in equations (A 1)-(A.9) are given by

$$
\begin{aligned}
A & \equiv-\sqrt{ }\left(\frac{3}{2}\right) a_{0} \equiv-\sqrt{ }\left(\frac{3}{2}\right) \gamma_{I} \gamma^{\prime}{ }_{I} / r^{3} \\
B & \equiv a_{0}^{2} \tau\left(\eta_{0}(\beta)+\frac{1}{3} \eta_{2}(\beta)\right), \\
C\left(L, L^{\prime} ; K, M\right) \equiv \frac{2}{3} a_{0}{ }^{2} \tau \eta_{2}(\beta) N\left(L, L^{\prime}\right) \times(-)^{K-M} & \\
& \times\left(\begin{array}{cccc}
2 & L & L^{\prime} \\
0 & -K & K
\end{array}\right) \times\left(\begin{array}{cccc}
2 & L & L^{\prime} \\
0 & -M & M
\end{array}\right)
\end{aligned}
$$

and

$$
\omega_{I} \equiv \gamma_{I} H_{0},
$$

where all terms are in angular frequency units of $\mathbf{s}^{-1}$.

\section{REFERENCES}

[1] Woessner, D. E., 1962, 7. chem. Phys., 36, 1 ; 1962, Ibid., 37, 646.

[2] Donskaya, I. S., 1975, Molec. Phys., 29, 1361.

[3] Wallach, D., 1975, F. chem. Phys., 47, 5258.

[4] Lambert, J. B., Nienhuis, R. J., and Keepers, J. W., 1981, Ang. Chem. (Engl), 20, 487.

[5] Abragam, A., 1961, The Principles of Nuclear Magnetism (Oxford University Press), Chap. VIII.

[6] Hwang, L. P., Anderson, C. F., and Friedman, H. L., 1975, F. chem. Phys., 62, 2098.

[7] Hwang, L. P., and Freed, J. H., 1975, F. chem. Phys., 63, 118.

[8] Freed, J. H., Bruno, G. V., and Polnaszek, C. F., 1971, Y. phys. Chem., 75, 3386.

[9] Wittebort, R. J., and Szabo, Attila, 1978, f. chem. Phys., 69, 1722.

[10] Campbell, R. F., Meirovitch, E., and Freed, J. H., 1979, F. phys. Chem., 83, 525.

[11] Campbell, R. F., and Freed, J. H., 1980, F. phys. Chem., 84, 2668. 